\title{
Antibacterial and Morphological Studies of Electrospun Silver-Impregnated Polyacrylonitrile Nanofibre
}

\author{
T. O. SIYANBOLA ${ }^{1 *}$, T. GURUNATHAN ${ }^{2}$, A. F. AKINSOLA ${ }^{3}$, \\ J. A. ADEKOYA ${ }^{1}$, A. A. AKINSIKU'1, O. ALADESUYI', S. RAJIV ${ }^{4}$, \\ S. MOHANTY ${ }^{5}$, T. S. NATARAJAN ${ }^{6}$ and S. K. NAYAK ${ }^{2}$ \\ 'Department of Chemistry, Covenant University, P. M. B. 1023, Ota, Ogun State, Nigeria. \\ ${ }^{2}$ Central Institute of Plastics Engineering \& Technology, Guindy, Chennai 600032, India. \\ ${ }^{3}$ Department of Chemistry, Ekiti State University, P.M.B. 5363, Ado-Ekiti, Nigeria. \\ ${ }^{4}$ Department of Chemistry, Anna University, Chennai-600025, India. \\ ${ }^{5}$ Physics Department, Indian Institute of Technology, Madras, Chennai-600036, India. \\ ${ }^{*}$ Corresponding author E-mail: tolu.siyanbola@ covenantuniversity.edu.ng \\ http://dx.doi.org/10.13005/ojc/320116 \\ (Received: January 29, 2016; Accepted: February 27, 2016)

\section{ABSTRACT} \\ Silver-impregnated polyacrylonitrile (PAN) nanofibre was prepared through electrospinning \\ process. Infra-red spectroscopy (FT-IR), X-ray powder diffraction (XRD), scanning electron microscopy \\ (SEM), Thermo gravimetric analyses (TGA/DTA) were used to characterize PAN and PAN/Ag \\ composites. The XRD results reflects decrease in the crystallinity of PAN as it is been modified with \\ Ag. Antibacterial activity of PAN/Ag was also investigated.
}

Key word: Antibacterial activity, Nanofibre, Silver, Polyacrylonitrile, Electrospinning, Composite.

\section{INTRODUCTION}

The undeniable contribution of electrospinning to nanotechnology development especially in the industrial and academic sectors cannot be over emphasized. It provides cheap, uncomplicated effective technology towards synthesizing nanofibre polymers with excellent properties such as flame retardation, enhanced dimensional stability, high porousity and specific surface area ${ }^{1-2}$. The high surface area property exhibited by nanofibre makes high degree of physical contact possible by creating sites for chemical reaction which is the basis for various modification of the nanofibre or trapping fine particle size material through physical entanglement thereby making filtration property of the material possible. Other areas of immense application includes tissue 
scaffold, material for dressing wounds, sensors, storage cells for hydrogen fuel cells, drug delivery systems, catalysis reaction and protective clothing etc $^{3-5}$. Typical electrospinning procedure involves the use of high voltage source that provides the required electric field on the polymer solution or melts which in turn jets out of the syringe. The dry jets nanofibre move towards an earmark opposite charge collector which capture the polymer fibre (Figure 1). The process is void of coagulation chemistries or high temperatures that can produce solid threads from solutions. The type of polymer solution and/ or spinneret modification will determine the type of nanofibre that is will be formed. Sometimes, the nanofibres can exhibit porous or core-shell morphology depending on the material undergoing spinning, nozzle to collector distance as well as its miscibility and rate of evaporation of the solvent involved 6 . Megelski et al., (2002) reported bead formation morphology in electrospun polystyrene fibre but on decreasing nozzle to collector plate distance but on increase the nozzle to collector plate distance, the ribbon shaped morphology is conserved ${ }^{7}$. It is however important to state that alteration of the distance between the feeding nozzle and the fibre collecting device gives more drying time to the electrospun fibres. Several base polymeric materials such as collagen, polylactideglycolide, poly(ethylene oxide), silk protein, cellulose derivatives and polyvinyl alcohol (PVA) have been used as precursors ${ }^{8}$.

The impregnation of inorganic nanoparticles into polymer matrix has been synthetically achieved recently ${ }^{10}$. Nanoparticles such as metal oxide, metal, metal sulphide, metal nitrate and so on. The internalization of nano-AgX into polymeric matrix offers the composite extensive industrial viabilities that combine features of organic and inorganic materials ${ }^{11-12}$. They provide composite materials having antimicrobial, optical and electrical applications ${ }^{10}$, catalyst and photochromic materials ${ }^{13-14}$.

\section{EXPERIMENTAL}

Polyacrylonitrile (PAN) and silver nitrate was purchased from Sigma Aldrich, India. DMF was obtained from Sisco Research Laboratory Private Limited, India.

\section{Solution preparation}

PAN solution and $\mathrm{PAN} / \mathrm{AgNO}_{3}$ solution were both separately prepared by dissolving them in DMF and respectively stirred continuously for 8 and 24 hours on a magnetic stirrer.

\section{Preparation of dispersed Ag nanoparticle in PAN nano-fibrous film}

A $10 \times 20 \mathrm{~mm}$ nano-fibrous film of PAN/ $\mathrm{AgNO}_{3}$ was place in a $50 \mathrm{~mL}$ beaker and $20 \mathrm{~mL}$ triple distilled water plus $1 \mathrm{~mL} \mathrm{~N} \mathrm{H}_{5} \mathrm{OH}$ aqueous solution was added to it. This reduces the silver ions in PAN nanofibrous film to silver at room temperature. After 30 minutes, the film was washed with distilled water and alcohol. After which, it was dried in a vacuum oven.

\section{Electrospinning of PAN/Ag}

Figure 1 shows the set-up used for electrospinnig of PAN/Ag. The polymer solution of PAN/Ag was taken into a $2 \mathrm{~mL}$ syringe connected to a capillary tip of $0.56 \mathrm{~mm}$ inner diameter. The positive electrode to which high voltage power supply is connected is wrapped with aluminium foil. Optimization of electric voltage was at $15 \mathrm{KV}$. Different polymer solutions concentrations were electrospun at $0.7 \mu \mathrm{L} / \mathrm{min}$ flow rate and $10 \mathrm{~cm}$ tip to collector distance. All experiments were carried out at $25^{\circ} \mathrm{C}$.

\section{Characterization \\ Fourier Transform Infrared Spectroscopy (FT-IR) analysis}

The functional groups present in the spun fibres were identified by Bruker 66V FT-IR spectrometer in the range $4000-400 \mathrm{~cm}^{-1}$.

\section{Scanning electron microscopy (SEM) analysis}

Eletrospun fibre mats were sputter coated with gold and the micro to nano-fibres morphology were observed on a high resolution scanning electron microscope (HRSEM). Scion image software was used analyzing the fibre diameters, orientation pattern and surface of the individual fibre.

\section{X-Ray diffraction (XRD)}

The X-ray diffraction measurements of nanofibre were performed on Rigaku Miniflex II instrument. The nanofibrous membrane was 
pressed inside the diffractometer sample holder and patterned data were collected in-situ.

\section{Thermal analysis}

The thermal stability of PAN and PAN/Ag nano-fibrous materials were ascertained by TGA/ DTA analysis. Samples weight ranging from 4-8 mg was placed in the platinum sample pans under a continuous nitrogen atmosphere with a flow of 25 $\mathrm{mL} / \mathrm{min}$ in a TGA/DTA model SDT 2600 instrument. The sample and its reference were heated at the rate $20^{\circ} \mathrm{C} / \mathrm{min}$. The phase changes and glass transition temperature of the nanofibrous Pan and PAN/Ag were measured on Perkin-Elmer differential scanning calorimeter (DSC-2).

\section{Antibacterial activity}

The standard LB plate method was used to evaluate the antibacterial activity of the $\mathrm{AgNO}_{3} /$ PAN precursor solution against gram negative Escherichia coli (E. coli). The ready-made nutrient agar was suspended in double distilled water (1000 $\mathrm{mL}$ ) and heated to boiling for 25 min until it dissolved completely; the medium and Petri dishes were autoclaved at pressure $15 \mathrm{Lb} / \mathrm{in}^{2}$. The medium was transferred into sterile Petri dishes under aseptic conditions in a laminar air flow chamber. When the medium in the plates had solidified, $0.5 \mathrm{~mL}$ (approx. $10^{6} \mathrm{CFU} / \mathrm{mL}$ ) of culture of test organism was inoculated and evenly spread over the agar surface with a sterile L-shaped rod. Embedded nanofibrous

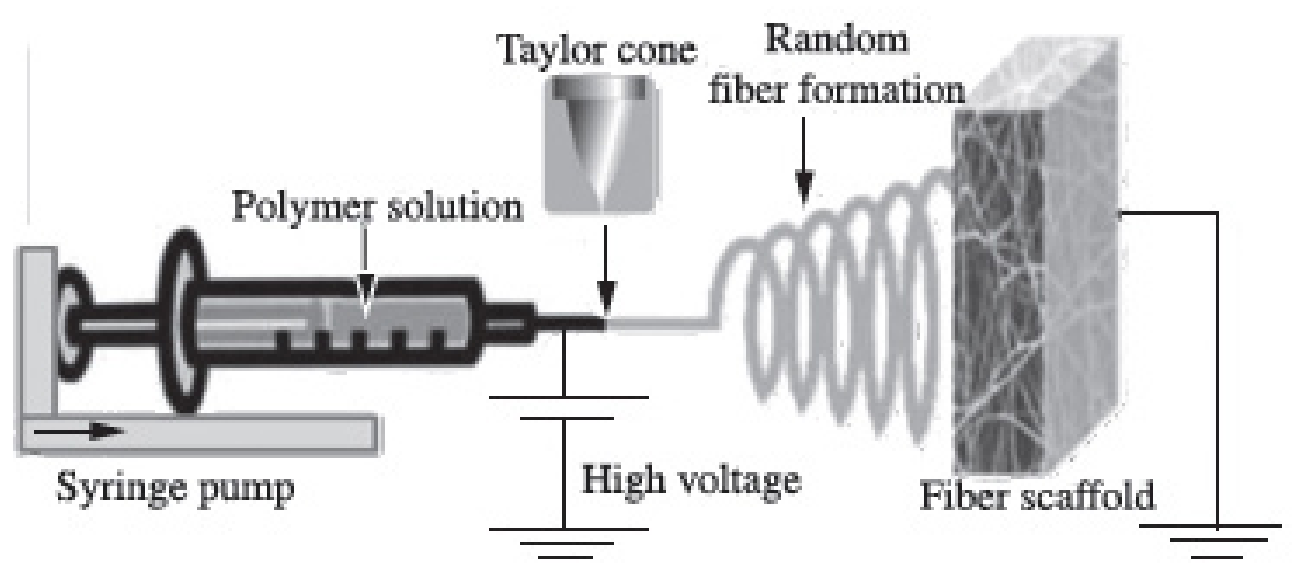

Fig. 1a: Electropinning operation (Source: Google image9)

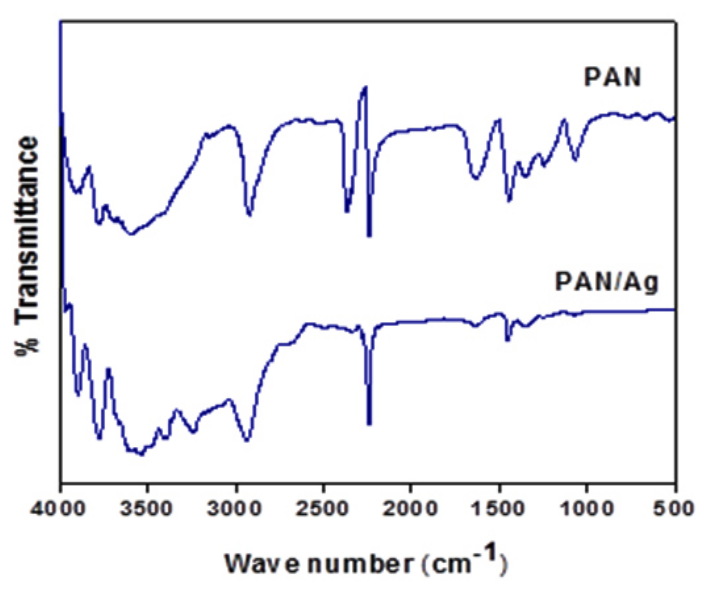

Fig.1b: Overlay FT-IR Spectra of PAN and PAN/Ag nanofibres

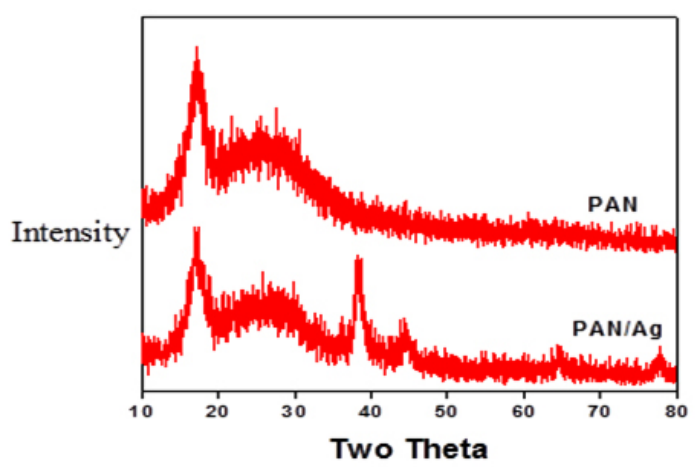

Fig. 2: XRD patterns of PAN and PAN/Ag nanofibres 
samples with $2 \mathrm{~cm} \times 2 \mathrm{~cm}$ (approx.) samples were cleaned with double distilled water and placed on the medium and incubated at $37{ }^{\circ} \mathrm{C}$ for 24 hours. The antibacterial activity was carried out based on the formation of an inhibition zone and loss of growth of organisms beneath and surrounding the films placed on the agar medium. Three replicates were used for each treatment ${ }^{15}$.

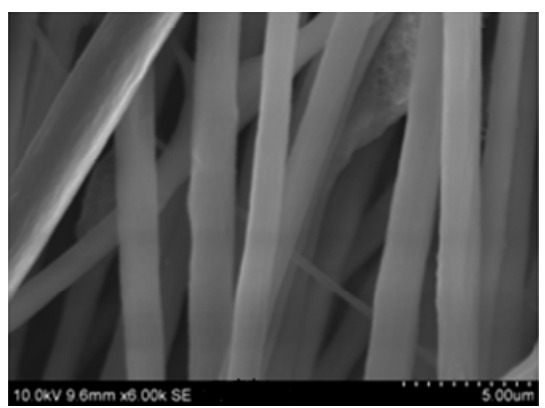

$3 a$

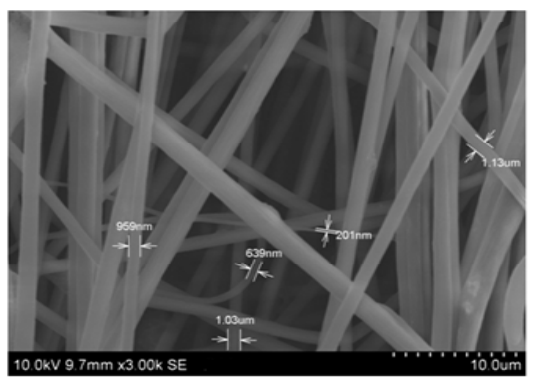

$3 b$

Fig. 3: SEM pictures of electrospun PAN nanofibre

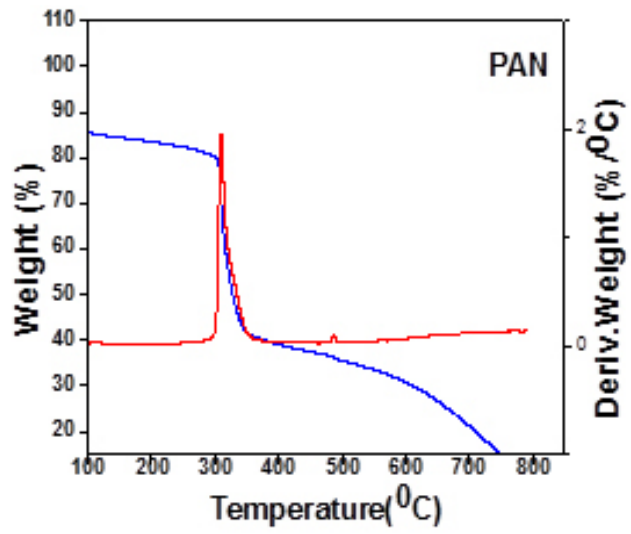

\section{RESULTS AND DISCUSSION}

Fourier Transform Infra-red Spectroscopy

It had been reported that electropinning process of composite solutions might lead to differences in the composition of the pre-spun solution and the nanofibres deposited ${ }^{12-13}$. In the light of this, the infra-red spectroscopy of deposited fibres was analyzed. The overlay FT-IR spectra of PAN and PAN/Ag are represented in Figure 1. PAN shows a characteristic stretching vibrational peak of nitrile group (-CN-) at $2244 \mathrm{~cm}^{-1}$. Also, stretching and bending vibrations of methylene $\left(-\mathrm{CH}_{2}-\right)$ functional group is seen at $2917 \mathrm{~cm}^{-1}$ and $1448 \mathrm{~cm}^{-1}$ respectively. As for PAN/Ag nanofibre, the peak at $1637 \mathrm{~cm}^{-1}$ is due to the oxidation of the PAN in air (as

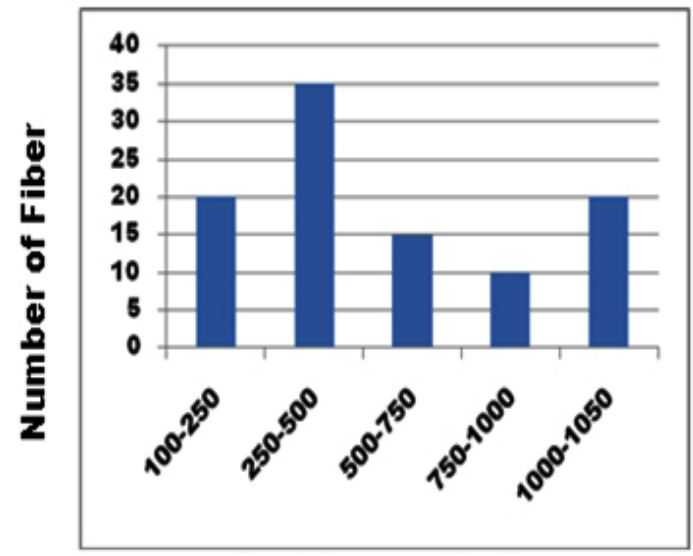

Fibre diameter (nm)

Fig. 4: Histogram peaks for SEM images electrospun PAN nanofibers

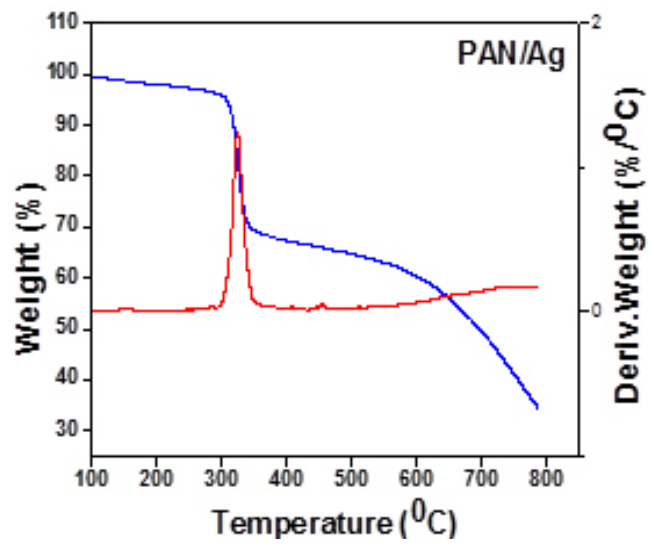

Fig. 5: TGA and DTA graph of PAN and PAN/Ag nanofibres 


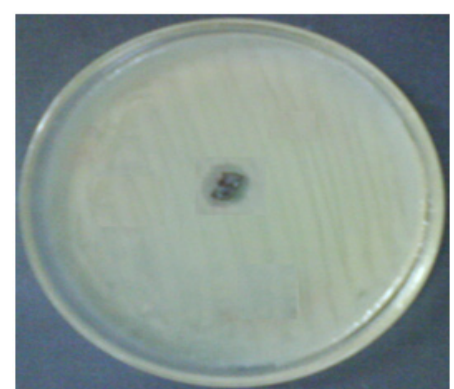

Fig. 6: Antibacterial activity of PAN/Ag nanofibre

obtained), which results in the formation of carboxyl $(\mathrm{C}=\mathrm{O})$ groups. The peaks at $1248 \mathrm{~cm}^{-1}$ and $1352 \mathrm{~cm}^{-1}$ are assigned to the aliphatic $-\mathrm{CH}$ - group vibrations of different modes in $\mathrm{CH}$ and $\mathrm{CH}_{2}$, respectively. The positions of these peaks in PAN/Ag composites nanofibre is observed to shift slightly lower values due to the interaction between PAN molecules and $\mathrm{Ag}$.

\section{X-ray diffraction analysis (XRD)}

The representative XRD patterns of PAN and PAN/Ag fibres are presented in figure 2. The PAN single unique diffraction peak is observed at $2 \theta=17^{\circ}$ while the modified electrospun nanofibre (PAN/Ag) shows other characteristic peaks at $38^{\circ}$, $44^{\circ}, 64^{\circ}$, and $77^{\circ}$ of $2 \theta$.

\section{Scanning electron microscopy (SEM)}

Figure 3 shows the SEM image of PAN electrospun nanofibre at $12 \mathrm{wt} \%$ concentration and at $5 \mu \mathrm{m}(3 \mathrm{a})$ and $10 \mu \mathrm{m}(3 \mathrm{~b})$ magnifications. The mean diameters of the fibres were found to be approximately $150 \mathrm{~nm}$ to $1 \mathrm{~nm}$ respectively. Figure 4 shows the histogram peaks for electrospun PAN nanofibres.
Thermogravimetric Analysis (TGA) and Differential Thermal Analysis (DTA)

The TGA and DTA thermograms of PAN and PAN/Ag fibres are presented in Figure 5. Both thermograms show evident weight loss peaks at 305-320 ${ }^{\circ} \mathrm{Cand} 600-740{ }^{\circ} \mathrm{C}$ in the TGA curves. The first weight loss is presumed to be caused by decarboxylation of the methylacrylate in PAN while, the second weight loss may be due to thermooxidative degradation of PAN macromolecular chains. For PAN/Ag nanocomposite, the first step degradation was observed at around $325^{\circ} \mathrm{C}$ whereas, that of PAN was at $309^{\circ} \mathrm{C}$. The PAN/Ag system is seen to be more thermally stable that PAN system.

\section{Antibacterial activity}

The antibacterial activity of PAN/Ag nanocomposite fibre is tested against Gram-negative Escherichia coli. The test was carried out with varying percentage of $\mathrm{Ag}^{+}$in PAN solution. As the percentage composition increases the zone of inhibition also increases (Figure 6). The formation of clear zone of inhibition around the PAN/Ag sample clearly shows that the $\mathrm{Ag}^{+}$ions were effective on the inhibition of bacterial growth.

\section{CONCLUSION}

Electrospun PAN and PAN/Ag nanofibres were characterized by FT-IR, XRD, SEM, TGA and DTA. Antibacterial activity of PAN/Ag was also evaluated. The SEM micrographs show $100 \%$ bead free nanofibre in $12 \mathrm{wt} \%$ ratio of dimethylformaldehyde (DMF). The FT-IR absorption peaks and XRD pattern of PAN/Ag clearly reveal the interaction of $\mathrm{Ag}$ with PAN molecules.

\section{REFERENCES}

1. Pham, Q. P.; Sharma, U.; Mikos, A. G. Tissue Engineering. 2006, 12, 1197-1211.

2. Park, J. H.; Karim, M. R.; Kim, I. K.; Cheong, I. W.; Kim, J. W.; Bae, D. G.; Cho, J. W.; Yeum, J. H. Colloid Polm. Sci. 2010, 288, 115-121.

3. Reneker, D. H.; Chun, I. Nanotechnology. 1996, 7, 216-223.

4. Li, D.; Xia, Y. Adv. Mater. 2004, 16, $1151-$
1170.

5. Son, W. K.; Youk, J. H.; Lee, T. S.; Park, W. H. Polym.2004, 45, 2959-2966.

6. Bazilevsky, A. V.; Yarin, A. L.; Megaridis, C. M. Langmuir. 2007,23, 2311-2314.

7. Megelski, S.; Stephens, J. S.; Chase, D. B.; Rabolt, J. F.Macromol.2002, 22, 8456-8466.

8. Won, H. P.; Lim, J.; Dong, I. Y.; Hudson, S. 
Polym. 2004, 45, 7151-7157.

9. Dhandayuthapani, B.; Yoshida, Y.; Maekawa, T.; Kumar, D. S.Mat. Res. 2011, 14,317-325.

10. Chan, Y.; Liu, H.; Zhang, Z. Appl. Mechan. \& Mater. 2010, 26-28, 159-162.

11. Mbhele, Z. H.; Salemane, M. G.; Van Sittert, C. Chem. Mater. 2003, 26, 5019-5024.

12. Kim, J. H.; Min, B. R.; Won, J.; Joo, S. H.; Kim, H. S.; Kang, Y. S. Macromol. 2003, 36, 61836188.

13. Currao, A.; Reddy, V .R.; Calzaferri, G. Chem. Phys. Chem. 2004, 5, 720-724.
14. Schurch, D.; Currao,A.; Sarkar,S.; Hodes,G.; Calzaferri, G.J. Phys. Chem. B. 2002,106, 12764-12775.

15. Siyanbola, T. O.; Sasidhar, K.; Rao, B. V. S. K.; Narayan, R.; Olaofe,O.; Akintayo,E. T.; Raju, K. V. S. N. J. Am. Oil Chem. Soc.2015, 92, 267-275.

16. Wongsasulak, S.; Kit, K. M.; McClements, D. J.; Yoovidhya, T.; Weiss, J.Polym. 2007, 48,448-457.

17. Kriegel,C.; Kit, K.M.; McClements, D.J.;Weiss, J. Polym. 2009,50, 189-200. 